\title{
„Sneak Preview“ in Lennep: Fensterpaten besuchen das Röntgen-Geburtshaus
}

Zahlreiche Neugierige nutzen am Sonntag die seltene Gelegenheit, noch vor der offiziellen Eröffnung einen Blick in das denkmalgeschützte Geburtshaus von Wilhelm Conrad Röntgen zu werfen. Zum bundesweiten Tag des offenen Denkmals am 9. September 2018 konnten sich die Besucherinnen und Besucher selbst einen Eindruck vom Stand der Renovierungs- und Umbauarbeiten verschaffen. Erstmals besuchten die Förderer, die eine Fensterpatenschaft übernommen haben, das Geburtshaus mit den neu eingesetzten Fenstern.

Remscheid-Lennep, 11.09.2018. „Gefunden! Ach, mein Fenster sieht aber wirklich schön aus!“ Begeistert erkundet ein gutes Dutzend Fensterpatinnen und -paten das kleine Fachwerkhaus in der Lenneper Innenstadt, in dem Wilhelm Conrad Röntgen geboren wurde. Die Patenschaft eröffnet Privatpersonen, Gruppen, Vereinen und Unternehmen die Gelegenheit, unmittelbar Teil eines einzigartigen Erinnerungsortes zu werden. Noch sieht es auf den drei Etagen nach viel Arbeit aus, denn aktuell stehen Lehmbauarbeiten auf dem Plan. So wie auch die Holzfenster originalgetreu nachgebaut wurden, werden auch die Innenwände nach alter Handwerkstradition mit Lehm und Stroh verputzt. „Sobald die Arbeiten abgeschlossen sind, können die letzten noch fehlenden Stromleitungen und die Fußböden verlegt werden“, berichtet die Architektin Sophie Welke vom aktuellen Stand.

\section{Ein lebendiges Denkmal}

Die Baustelle im Erdgeschoss wird aber bald Geschichte sein - denn im Frühjahr 2019 eröffnet dort eine Publikumsausstellung zum Leben von Wilhelm Conrad Röntgen. „Die Ausstellung widmet sich ganz der Person Röntgens und seiner Lebensleistung. In einer Schatzkammer werden wir auch ausgewählte Originale zeigen können, beispielsweise Briefe, die von einflussreichen Persönlichkeiten an Röntgen geschrieben wurden“, erläutert Dr. Uwe Busch, Direktor

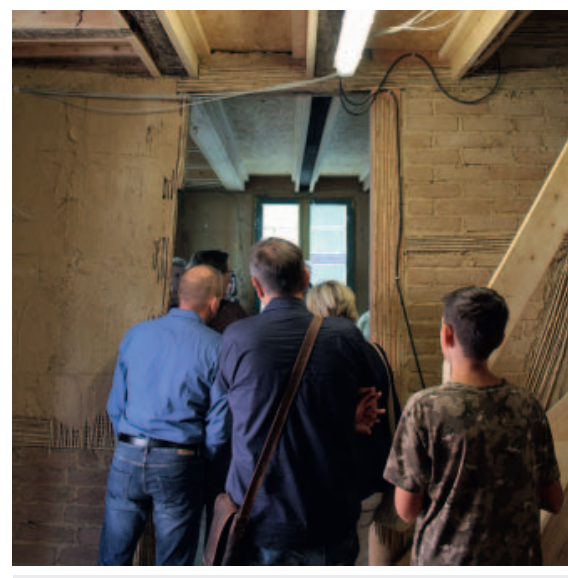

Hereinspaziert: Viele Neugierige machten sich ein Bild von den Baufortschritten im Röntgen-Geburtshaus. @ Deutsche Röntgengesellschaft e. V.

des Deutschen Röntgenmuseums. Die öffentliche und kostenfrei zugängliche Ausstellung soll dazu beitragen, dass das Haus zu einem lebendigen Denkmal wird und von Besucherinnen und Besuchern aus 


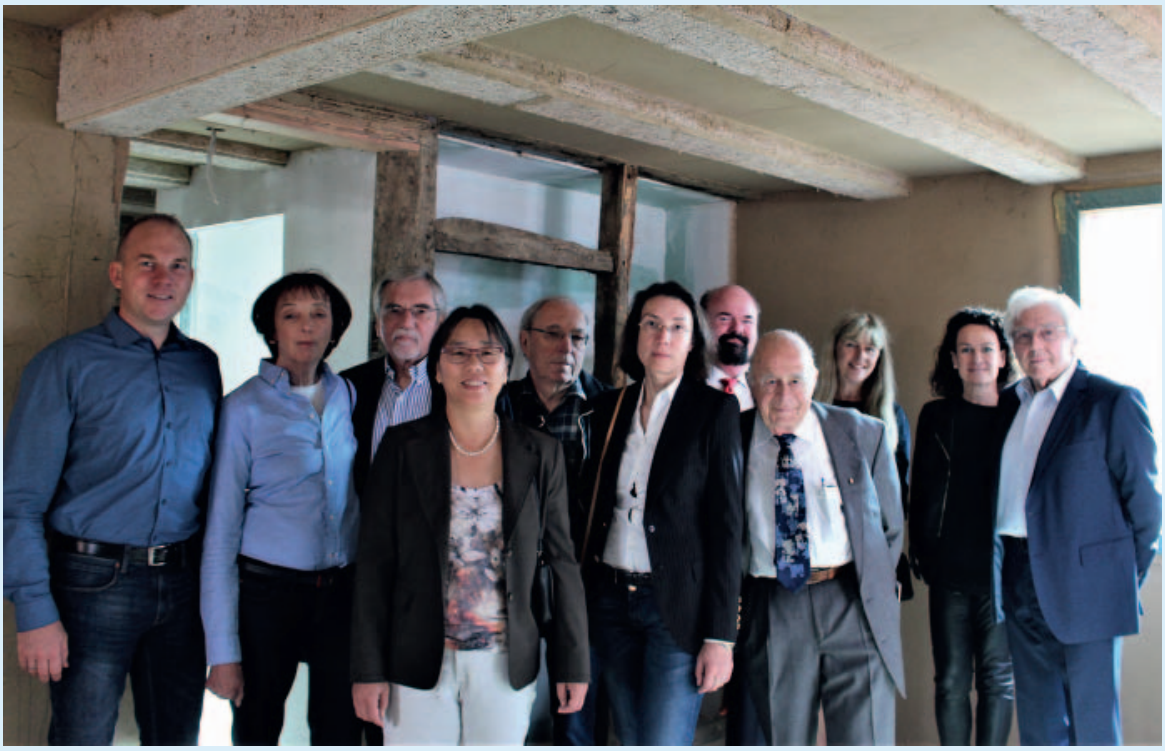

Zum Tag des offenen Denkmals 2018 besuchten die Fensterpaten erstmals „ihre“ Fenster im Röntgen-Geburtshaus. @ Deutsche Röntgengesellschaft e. V.

der Region, Deutschland und der Welt genutzt und erlebt werden kann.

\section{Neues Patenschafts- programm geplant}

Die Deutsche Röntgengesellschaft e. V. erwarb das denkmalgeschützte Gebäude 2011 von der Stadt Remscheid. Seither hat sie es sich zur Aufgabe gemacht, den Ursprungsort des weltberühmten Wissenschaftlers umfassend zu sanieren und zu neuem Leben zu erwecken. Getragen, begleitet und finanziert wird der zukünftige Treffpunkt von den rund 8500 Mitgliedern der Deutschen Röntgengesellschaft e. V., zahlreichen Bürgerinnen und Bürgern sowie Unternehmen aus der Region durch Patenschaften oder Geldspenden. Für die rund 20 Fenster fanden sich Patinnen und Paten aus Remscheid, Deutschland und der ganzen Welt. Da nun aber noch Mittel fehlen, um den Ausbau der ersten Etage als kleine Bibliothek und Konferenzraum voranzutreiben, ist ein neues Patenschaftsprogramm in Planung. Interessierte können beispielsweise für einzelne Möbel des Tagungsraumes eine Patenschaft übernehmen, oder für Bibliotheksschränke, in denen künftig wertvolle Bücher aus dem Bestand des Röntgenmuseums ihren Platz finden sollen.

\section{Geburtshaus ein zentraler Bestandteil des Jubiläumsjahrs 2020}

Am 8. November 1895 entdeckte Prof. Dr. Wilhelm Conrad Röntgen im Physikalischen Institut der Julius-Maximilians-Universität Würzburg die nach ihm benannten Strahlen, denen er zunächst den Namen X-Strahlen gab. Seine Entdeckung einer völlig neuen Art von Strahlen jährt sich 2020 zum 125sten Mal. Der am 27. März 1845 in Remscheid-Lennep geborene erste Nobelpreisträger für Physik würde im Röntgenjahr 2020 seinen 175. Geburtstag begehen. Um dieses bedeutsame Kapitel deutscher Wissenschaftsgeschichte einem Fachpublikum und der breiten Öffentlichkeit nahe zu

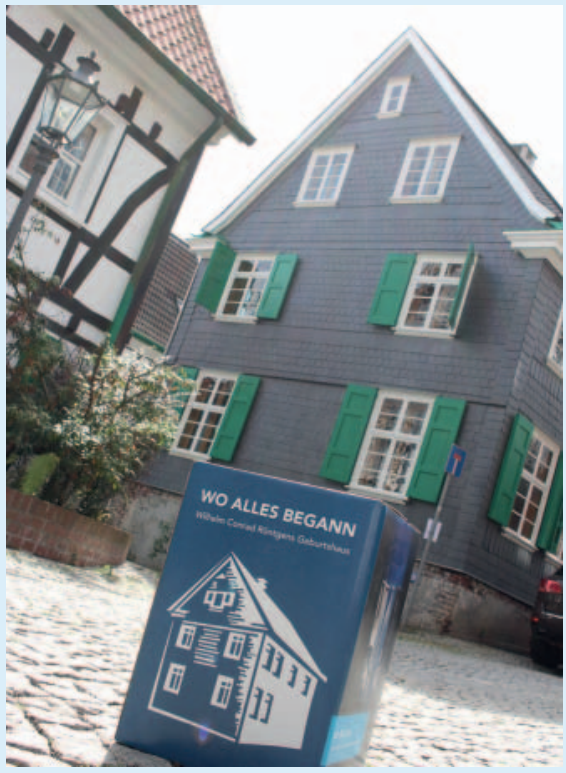

Bei schönsten Sonnenwetter präsentierte sich das Geburtshaus zum Tag des offenen Denkmals 2018 mit neuen Fenstern und renovierter Schieferfassade. (C) Deutsche Röntgengesellschaft e. V.

bringen, haben sich Universitäten, Hochschulen, Städte, Gesellschaften, Verbände und Institutionen zusammengeschlossen, die sich dem Erbe Wilhelm Conrad Röntgens verpflichtet fühlen und gemeinsam das Jahr 2020 als „Röntgen-Jahr“ gestalten wollen. Dem Geburtshaus von Wilhelm Conrad Röntgen kommt hierbei eine tragende Rolle zu. Während die Publikumsausstellung im Erdgeschoss bereits im Frühjahr 2019, unmittelbar vor dem 100. Deutschen Röntgenkongress (20. Mai bis 1. Juni 2019 in Leipzig) fertiggestellt sein wird, ist seine feierliche Eröffnung für das Jahr 2020 geplant.

Weitere Informationen rund um das Röntgen-Geburtshaus und die Patenschaften finden Interessierte unter www.roentgen-geburtshaus.de. 